\title{
Appointment of New Associate Editor and Editorial Board Members
}

Perspectives on Behavior Science welcomes a new Associate Editor:

David P. Jarmolowicz, University of Kansas

And new Editorial Board members:

John Borrero, $U M B C$

José E. Burgos, University of Guadalajara

Traci M. Cihon, University of North Texas

Anthony DeFullio, Western Michigan University

Iser G. DeLeon, University of Florida

Ronnie Detrich, Wing Institute

Wendy Donlin Washington, University of North Carolina Wilmington

Claudia Drossel, Eastern Michigan University

Tim Hackenberg, Reed College

Marta Hübner, Universidade de São Paulo

Elizabeth Kyonka, University of New England

Jack McDowell, Emory University

Jonathon R. Miller, University of Colorado School of Medicine

Suzanne H. Mitchell, Oregon Health \& Science University

Nancy A. Neef, The Ohio State University

Christopher Podlesnik, Florida Institute of Technology

Ingunn Sandaker, Oslo Metropolitan University

Claire C. St. Peter, West Virginia University 